Ces trois dernières décennies ont vu un bouleversement complet de la biologie structurale grâce à l'utilisation du rayonnement synchrotron. De nombreuses structures de macromolécules biologiques, inconnues jusqu'à ce jour, ont ainsi été déterminées.

L'automatisation des mesures de diffraction et le développement des techniques d'analyse et de modélisation ont facilité l'exploitation de ce nouvel outil.

Ces études structurales ont ainsi pu donner accès aux détails du fonctionnement des protéines et des molécules médicamenteuses qui interagissent avec elles, par exemple dans les combats contre les cancers ou le VIH, et ont influencé le développement de nouvelles thérapies.

\section{Le rayonnement synchrotron : comprendre la relation structure-fonction des macromolécules biologiques}

Matthew W. Bowler ${ }^{(1,2,3)}$ (mbowler@embl.fr) et Didier Nurizzo ${ }^{(3)}$

(1) European Molecular Biology Laboratory, 6 rue Jules Horowitz, BP 181, 38042 Grenoble

(2) Unit of Virus Host-Cell Interactions, UJF-EMBL-CNRS, UMI 3265, 6 rue Jules Horowitz, 38042 Grenoble Cedex 9

(3) Structural Biology Group, European Synchrotron Radiation Facility, 6 rue Jules Horowitz, 38043 Grenoble

\section{Le synchrotron} et la biologie structurale

Les protéines, polymères complexes d'acides aminés, sont impliquées dans l'ensemble des fonctions vitales des organismes vivants, allant de la digestion des aliments à la contraction des muscles en passant par la détection de la lumière, par exemple. Depuis la détermination en 1958, en utilisant le rayonnement X d'une anode tournante, une étude qui représentait alors l'œuvre de toute une vie [1] s'est réduite maintenant à quelques heures dans les cas les plus simples. de la première structure de la myoglobine
Quelles propriétés font du synchrotron un outil puissant pour l'étude structurale des protéines? Nous pouvons en évoquer trois : (i) un flux de photons jusqu'à 100000 fois plus élevé que celui d'une anode tournante, (ii) focalisé sur une surface le plus souvent plus petite que la taille du cristal lui-même, et (iii) la possibilité de faire varier la longueur d'onde du faisceau monochromatique, qui permet de résoudre le "problème des phases " (voir ci-après). De plus, le rayonnement synchrotron offre au biologiste deux méthodologies complémentaires et leurs techniques associées, pour déterminer la structure des protéines : la cristallographie des macromolécules (MX) et, pour l'étude

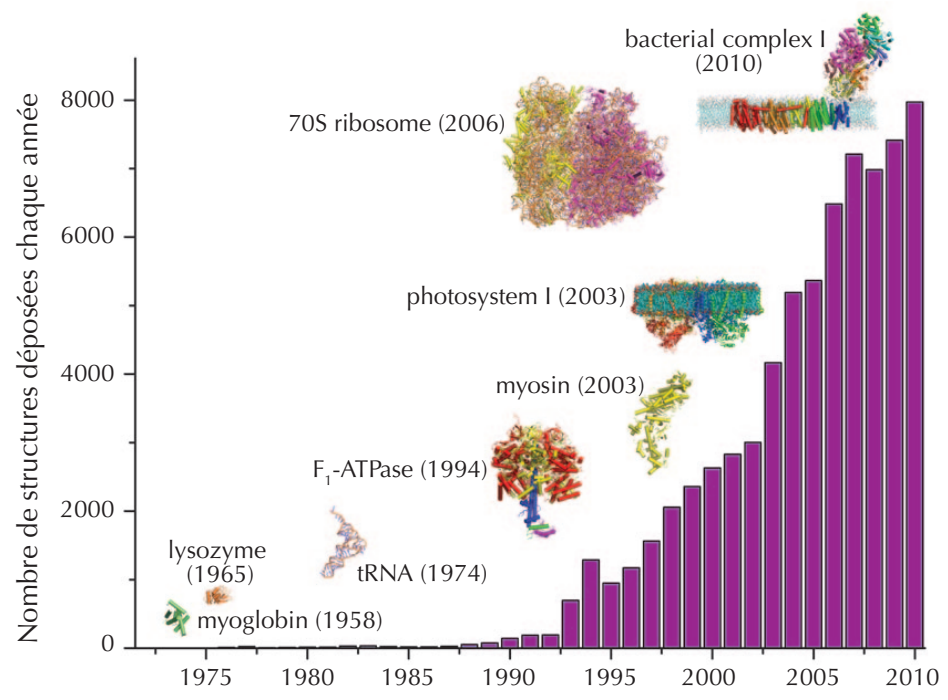

1. Statistiques indiquant le nombre de structures de protéines, d'ADN et d'ARN, déposées à la Protein Data Bank (PDB) chaque année depuis sa création en 1971 [2]. Les premières lignes de lumière de synchrotron ont été mises à la disposition des biologistes au début des années 1990, et l'automatisation véritable de certaines d'entre elles date du début des années 2000. Les schémas de macromolécules illustrent la complexité croissante des structures résolues. 


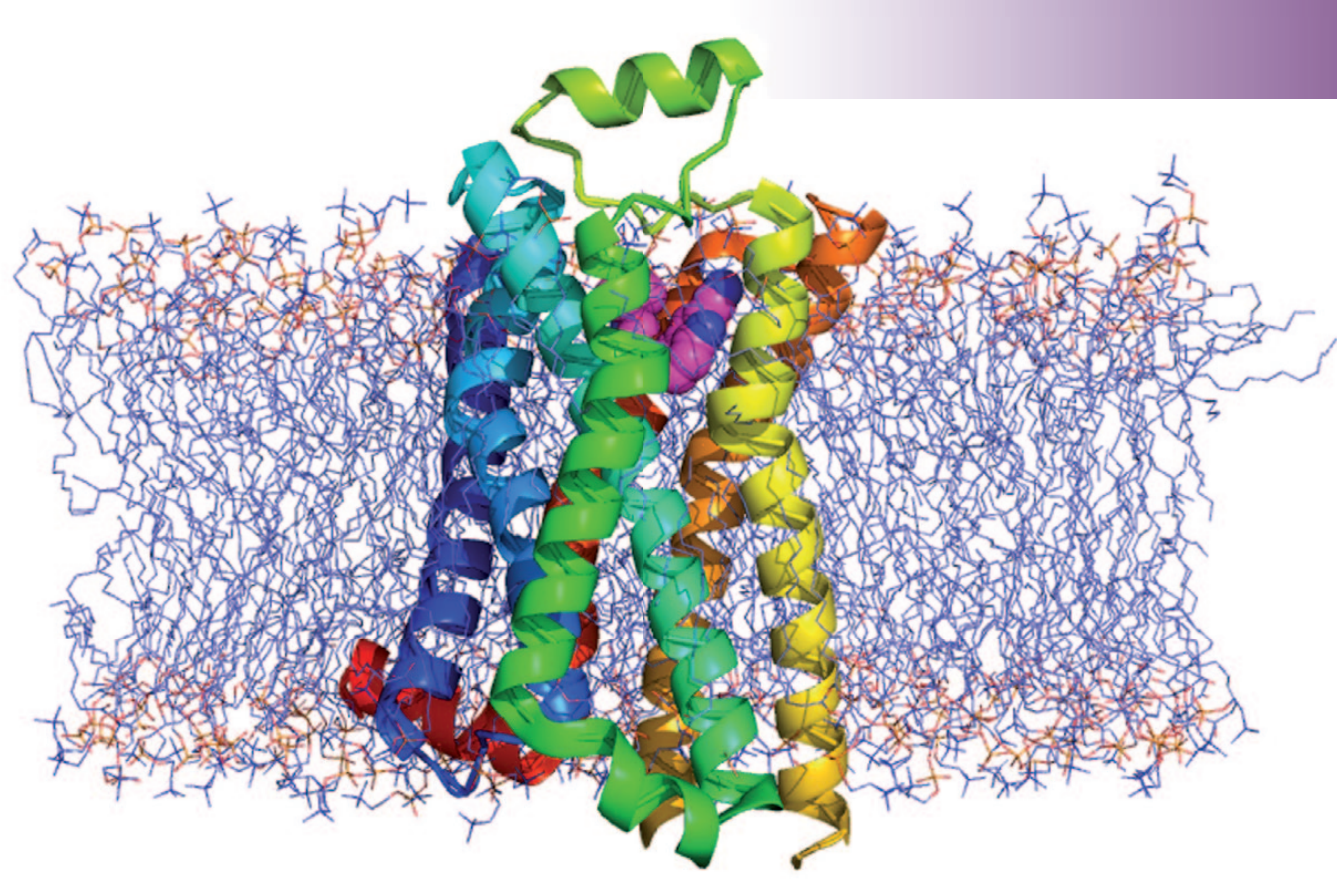

Représentation schématique du squelette carboné de la protéine $\beta_{1} A R$, montrant son repliement structural tridimensionnel au sein de la bicouche lipidique d'une membrane cellulaire (représentée en traits fins). La variation de couleur en arc-en-ciel suit la séquence peptidique. Les sphères violettes montrent comment une molécule agoniste ${ }^{(2)}$ de l'adrénaline vient se fixer à l'intérieur de la protéine. Le signal est transmis à travers la membrane par l'intermédiaire des hélices (voir p. 50).

des protéines en solution, la diffusion aux petits angles (SAXS), qui donne des informations sur leurs mouvements. Les nouvelles technologies liées à la recombinaison génétique d'ADN ouvrent la porte à l'expression ${ }^{(b)}$ de quasiment toutes les protéines, en quantité et en qualité, pour mener à bien leur étude structurale.

C'est grâce à ces avancées technologiques que le nombre de structures résolues a explosé (fig. 1), passant de sept lors de la création en 1971 de la banque de données Protein Data Bank (PDB), à 70000 de nos jours [2]. La connaissance de ces structures est primordiale, car dans la plupart des cas, elle a permis une meilleure compréhension du fonctionnement intrinsèque des protéines au niveau atomique, avec les conséquences médicales, industrielles et technologiques qui en découlent.
Dans cet article, nous présentons d'abord le déroulement d'une expérience de cristallographie macromoléculaire, puis quelques études de pointe réalisées au synchrotron et les implications de leurs résultats pour la recherche en biologie.

\section{Techniques de cristallographie macromoléculaire en biologie}

L'intérêt majeur de la cristallographie macromoléculaire est de donner une information structurale à un niveau de détail atomique sur les protéines et/ou l'ADN cristallisés. Mais, comme son nom l'indique, cette méthode est subordonnée à l'obtention de cristaux protéiques. Cette étape représente en ellemême un énorme travail qui peut, dans certains cas, rester infructueux pendant de longues années. La production de quantités importantes (de l'ordre du milligramme) de protéines en solution quasi pure est souvent nécessaire, et les cristaux obtenus sont en général de petite taille (de quelques à une centaine de micromètres). Ces cristaux sont préparés par concentration lente d'une liqueur mère (fig. 2a). Ils contiennent en moyenne $50 \%$ d'eau et sont très sensibles aux rayons $\mathrm{X}$ intenses; ils doivent donc être protégés, en les congelant à la température de l'azote liquide sous forme de glace vitreuse pour éviter la diffraction de la glace elle-même. Pour une manipulation plus aisée, les cristaux sont sortis de leur liqueur mère et montés dans une boucle ajustée avant d'être plongés dans l'azote liquide. Durant la totalité du temps de l'expérience de diffraction, le cristal sera maintenu sous
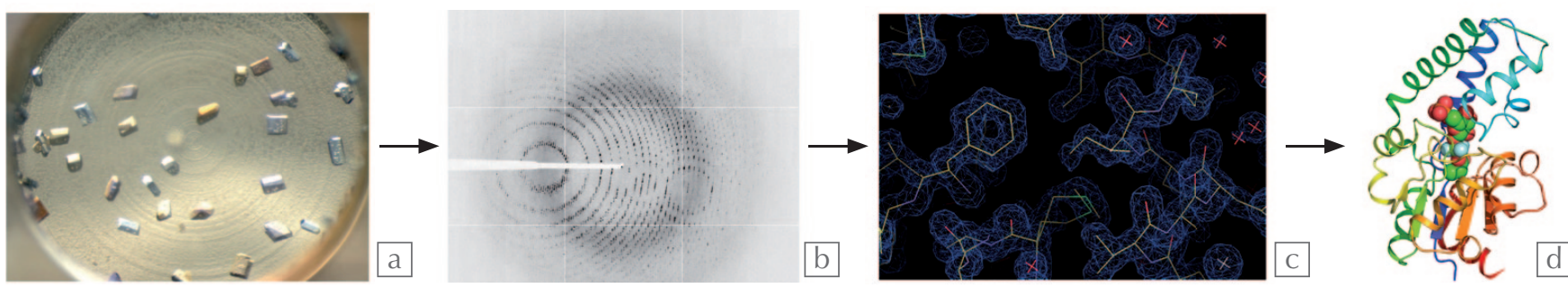

2. Les différentes étapes de l'obtention d'un modèle moléculaire de protéine.

(a) Les cristaux sont habituellement produits en concentrant une solution protéique pure en présence de sels et/ou de polymères, qui agissent comme agents précipitants (la taille des cristaux de protéine est généralement comprise entre 1 et $500 \mu \mathrm{m}$ ).

(b) Cliché de diffraction obtenu par oscillation du cristal autour d'un axe, dans un domaine angulaire de $0,5^{\circ}$. En moyenne, une oscillation totale de $90^{\circ}$ est nécessaire à l'obtention d'un modèle moléculaire.

(c) Carte de densité électronique (maillage bleu), calculée par transformée de Fourier des intensités collectées en (b) ; on en déduit le modèle moléculaire, représenté par ses segments interatomiques (avec les atomes de carbone, d'oxygène, d'azote et de soufre en jaune, rouge, bleu et vert respectivement).

(d) Une fois le modèle complet construit, le repliement général de la protéine est déterminé, et les positions des ligands et/ou substrats potentiellement présents (représentés sur la figure par des sphères) sont définies précisément. On peut alors en déduire la fonction et l'activité de la protéine au niveau atomique. 


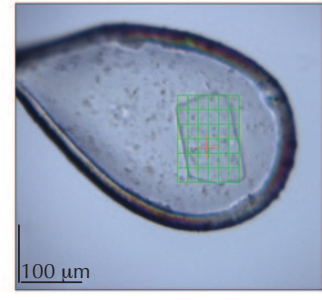

$\mathrm{a}$

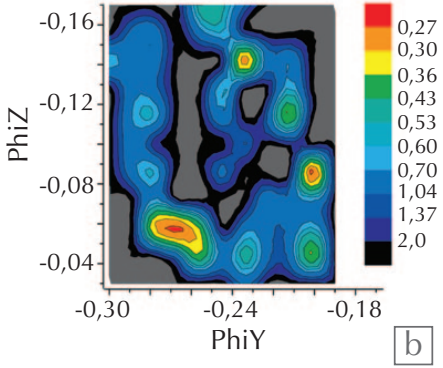

b

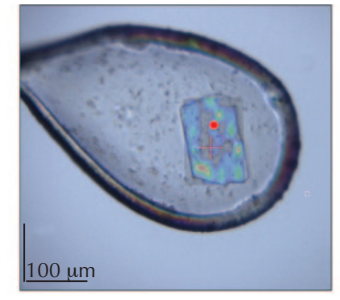

C
3. Cartographie par diffraction d'un cristal de $\beta_{1} A R$.

(a) La surface du cristal est quadrillée virtuellement, chaque point d'intersection correspondant à un point de test de diffraction par pas de $15 \mu \mathrm{m}$ dans les deux dimensions.

(b) La carte de qualité de diffraction montre une grande variabilité sur la face du cristal.

(c) Cette carte est alors superposée au cristal lui-même afin d'aider à choisir rapidement les régions cristallines propres à une collection de données de grande qualité. Dans ce cas, la limite de résolution est de 2,78 A.

\section{》〉}

flux d'azote à la température de $100 \mathrm{~K}$. Une fois monté sur le diffractomètre, on évalue la "qualité " de son spectre de diffraction, et l'expérimentateur choisit le ou les meilleurs échantillons. Il procède alors à la collecte des données en prenant entre 30 et 2000 images par cristal, par simple rotation du cristal autour d'un axe unique. Chaque image est une collection de taches de diffraction (fig. 2b), qui seront individuellement analysées pour déterminer leur intensité et l'erreur de mesure.

La reconstruction d'un modèle protéique tridimensionnel à partir des clichés de diffraction d'un cristal se heurte au problème de la détermination de la phase de l'onde diffractée : la mesure des intensités des taches de diffraction ne fournit que le module du facteur de structure complexe du cristal. Dans le passé, on réglait ce problème en insérant dans le cristal protéique des atomes lourds (platine, mercure...) qui venaient modifier son facteur de structure, permettant ainsi de remonter à l'information sur les phases par comparaison avec le cristal natif. De nos jours, les phases sont obtenues en faisant la différence entre les clichés de diffraction mesurés pour plusieurs énergies du faisceau monochromatique, ce qui n'est possible que grâce à l'utilisation d'un rayonnement synchrotron. Dans la région de fluorescence d'un atome prédéfini (soufre, calcium, zinc...) présent dans le cristal, la loi de Friedel reliant une paire de taches de diffraction symétriques $(h, k, l)$ et $(-h,-k,-l)$ ne s'applique plus, et la différence d'intensité entre les deux taches permet par analyse statistique de retrouver les phases. Une autre méthode, plus simple, appelée remplacement moléculaire, utilise les phases d'un modèle déjà résolu et proche du cristal étudié.

Une fois les phases et les intensités connues, une carte de densité électronique peut être calculée par transformée de Fourier dans l'unité asymétrique qui est le volume non réductible par lequel le cristal peut être reconstruit grâce à sa symétrie intrinsèque (fig. 2c). La carte de densité électronique doit ensuite être interprétée par le scientifique lui-même dans les cas les plus extrêmes; mais cette étape peut aussi être automatisée, afin d'y placer le modèle atomique d'acides aminés qui permettra une compréhension au niveau atomique de la fonction de la macromolécule étudiée (fig. 2d).

Ce processus, ainsi résumé en quelques lignes, peut rencontrer de nombreux problèmes. L'un d'entre eux, et non le moindre, est la variabilité de la qualité de diffraction des cristaux. Le plus souvent, il faut examiner des centaines de cristaux avant de pouvoir procéder à une collecte de données. Cette problématique a été abordée à l'ESRF sous l'angle de l'automatisation [3]. Tout d'abord, on utilise en routine des chargeurs d'échantillons, robots présents sur la ligne de lumière, qui vont automatiquement monter sur le diffractomètre les échantillons maintenus dans l'azote liquide, supprimant de ce fait une intervention manuelle souvent longue et difficile. Ces robots sont capables de charger et décharger jusqu'à 300 échantillons par jour. Ensuite, un logiciel d'analyse qualitative classe rapidement les cristaux par ordre de qualité, en prenant en compte différents paramètres (mosaïcité, limite de diffraction, symétrie...). Enfin, une interface d'utilisation aisée permet une meilleure gestion de l'expérience.

Grâce à l'utilisation de faisceaux de petites tailles comparées à celles des cristaux eux-mêmes, couplée à cette automatisation, l'expérimentateur peut, en routine, déterminer au sein d'un cristal les régions qui donneront des données de diffraction de haute qualité, et mener précisément son expérience dans ces régions (fig. 3). Cette nouvelle possibilité de scruter automatiquement de multiples positions dans un cristal et de visualiser immédiatement les résultats de l'analyse a été la clé de la réussite de la détermination de la structure des G protein coupled receptors (GPCRs).

\section{La première structure de GPCRs}

Si vous comptez sur votre petit café matinal pour bien commencer la journée, pensez que ce sont les GPCRs qui vous donnent de l'élan. Ces petites protéines, enfichées dans les membranes cellulaires, se lient aux molécules qui passent dans leur voisinage et développent une réponse physiologique adéquate. Elles seront, par exemple, responsables de la réaction de la rétine à la lumière, de la réponse olfactive aux odeurs, mais elles se lient également aux hormones et aux neurotransmetteurs qui règlent l'humeur ou bien la fréquence des battements de cœur. La moitié des médicaments actuellement sur le marché, notamment ceux utilisés en traitement de la migraine, de l'asthme et des maladies cardiaques, visent les voies biochimiques de signalisation que stimulent les GPCRs. L'homme compte près de 800 GPCRs différentes, dont 450 sont olfactives. Les 350 autres ont un fort intérêt pharmaceutique et sont identifiées par différentes sociétés comme cibles de médicaments potentiels.

La famille des GPCRs compte parmi ses membres le récepteur adrénergique ${ }^{(c)}$ $\beta_{1}$ AR, modulant la fonction cardiaque. Il a fallu plus de 18 ans pour obtenir les premiers cristaux de $\beta_{1} \mathrm{AR}$, car les protéines membranaires ne sont souvent produites qu'en faible quantité et sont très instables une fois sorties de leur environnement cellulaire. Leur purification se fait en présence de détergents. De plus, les protéines sont en agitation 
continuelle, même en présence de leur molécule cible. Dans ces conditions, la cristallogenèse est un véritable défi, mais qui finalement a pu être surmonté.

L'étape suivante a été la collecte de données sur ces mêmes cristaux. L'utilisation de faisceaux microfocalisés (disponibles sur les lignes de lumière ID13 et ID23-2 de l'ESRF), dont le diamètre est réduit à environ $5 \mu \mathrm{m}$, a été un des atouts majeurs de la réussite de ce projet. Un millier de cristaux de $\beta_{1} \mathrm{AR}$ ont été successivement testés afin d'en identifier un qui a finalement permis de collecter un jeu de données complet à une résolution de $2,7 \AA$, en combinant trois positions différentes à l'intérieur de ce cristal (protéine complexée avec un médicament). Le modèle ainsi obtenu [4] révèle des détails précis concernant la fonction de cette protéine et ses interactions avec son médicament (voir la figure en haut de la page 49). Cette découverte a eu un énorme impact sur la recherche pharmaceutique et médicale.

\section{Du gène à la protéine : le ribosome}

L'acide désoxyribonucléique (ADN) contient l'information génétique (génome codant) pour l'ensemble des protéines responsables de la majorité des fonctions biologiques du règne vivant. La première étape de traduction du gène consiste en une transcription de cette information, présente dans le noyau de la cellule, en un acide ribonucléique messager $\left(\mathrm{ARN}_{\mathrm{m}}\right)$, qui peut traverser la membrane nucléaire. Une machine nucléaire complexe, le ribosome, situé à l'intérieur du cytoplasme de la cellule, est capable alors de lire cette information génétique et de la traduire en une séquence d'acides aminés : la protéine. Les ribosomes sont eux-mêmes principalement formés d'ARN, complexés à quelques protéines. Ils comportent deux sous-unités structurelles, une grande et une petite (fig. $4 \mathrm{a}$ ). La petite sous-unité est responsable de la synthèse du peptide $^{(\mathrm{d})}$. Comme source d'acides aminés, le ribosome utilise des ARN de transfert $\left(\right.$ ARN $\left._{t}\right)$, qui sont des complexes covalents entre un acide aminé et un ARN. Chaque $\operatorname{ARN}_{t}$, porteur d'un des 20 acides aminés, reconnait spécifiquement un des 64 codons (triplets de bases nucléiques) du génome. La séquence protéique est donc dictée par la séquence de ce même génome. L'ARN $N_{t}$ et son acide aminé couplé sont délivrés au ribosome par une protéine appelée Facteur d'élongation Tu (FE-Tu). Cette protéine a une interaction forte avec l'ARN $_{t}$ et ne le libérera au polypeptide ${ }^{(\mathrm{d})}$ en cours d'élongation qu'après reconnaissance du codon spécifique.

Cette phase cruciale de la traduction fait toujours l'objet de travaux de recherches, d'autant plus que le FE-Tu est cible potentielle de nombreux antibiotiques. Plusieurs hypothèses expliquant cette étape ont été proposées dans le passé, mais aucun modèle atomique unique ne pouvait alors répondre à l'ensemble des questions soulevées concernant la
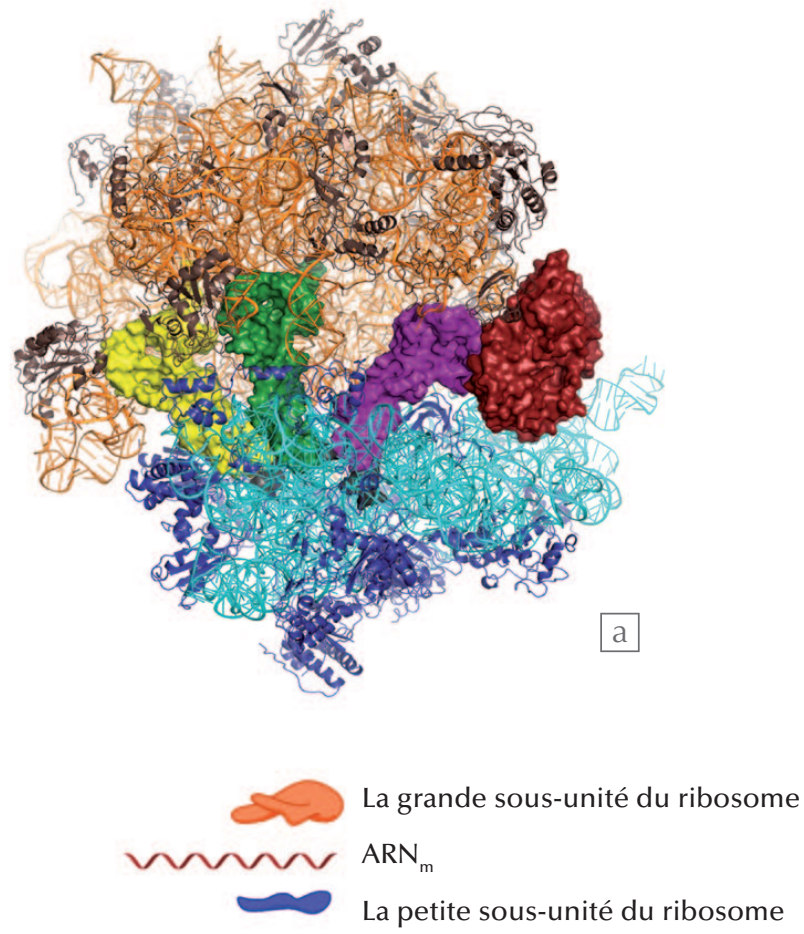
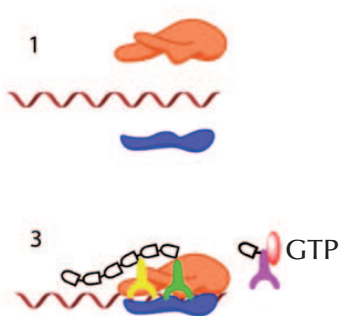

5
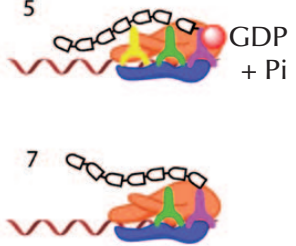
Facteur d'élongation
Tu (FE-Tu) avec GTP

FE-Tu avec GDP
2

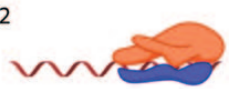

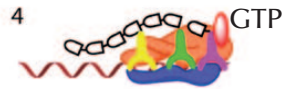
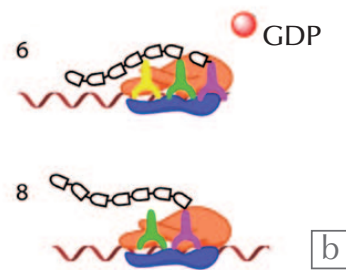

9. ARN de transfert (ARNt)

Polypeptide (protéine)
en cours d'élongation

4. Traduction d'une protéine par le ribosome.

(a) Structure du ribosome, formé d'une grande sous-unité (en haut, couleur or), et d'une petite (en bas, couleur bleue). Les protéines sont en couleurs foncées, l'ARN en couleurs claires. Le ribosome est en complexe avec le FE-Tu (en rouge, à droite) et l'ARN $($ (unités jaune, vert et magenta). La protéine FE-Tu empêche l'entrée de l'ARN ${ }_{t}$, encore lié à son acide aminé, tant que le codon n'est pas reconnu.

(b) Schéma de la traduction d'une protéine (code couleur identique à la figure a). 1. L'ARN ${ }_{m}$, copie de la séquence du gène ADN, est transféré du noyau vers le cytoplasme. 2. La petite et la grande sous-unité du ribosome se lient à l'ARN $\mathrm{m}_{\mathrm{m}}$ pour former trois sites de fixation de l'ARN $\mathrm{tà} \mathrm{I'ARN}_{\mathrm{m}}$. 3. L'anticodon de l'ARN $\mathrm{L}_{\mathrm{t}}$ se fixe au codon de l'ARN $\mathrm{m}_{\mathrm{m}}$ et la séquence protéique s'allonge par formation d'une liaison peptidique. 4. L'arrivée de l'ARN $\mathrm{t}_{\mathrm{t}}$ dans le site actif est fortement contrôlée : chaque ARN $\mathrm{A}_{\mathrm{t}}$ est d'abord activé par liaison avec la protéine FE-Tu ; si l'anticodon ne peut s'apparier au codon, le FE-Tu ne peut être relâché et l'ensemble est alors expulsé. 5. Si la paire codon-anticodon est correcte, le GTP est hydrolysé en GDP, et FE-Tu est libéré de I'ARN t puis relâché. 6. L’ARN t peut alors entrer dans le ribosome pour : 7. allonger la chaîne polypeptidique. 8. Le ribosome s'avance jusqu'au prochain codon de l'ARN $\mathrm{m}_{\mathrm{m}}$ pour un nouveau cycle. 


\section{>>}

quasi-absence d'erreurs entre l'information génomique et la séquence en acides aminés (fig. 4b).

Le groupe du Dr Venki Ramakrishnan du laboratoire de biologie moléculaire de Cambridge (Royaume-Uni) a été le premier à cristalliser le complexe formé entre le ribosome, le FE-Tu et l'ARN $\mathrm{N}_{\mathrm{t}}$ lié à son acide aminé. Ce complexe a été stabilisé en utilisant un antibiotique, la kirromycine, qui bloque la libération de l'ARN $N_{t}$. La très grande taille du complexe instable ainsi formé ( $300 \AA$ dans sa plus grande dimension) engendre une grande variabilité de qualité des différents cristaux produits, qui ont une taille moyenne de $500 \mu \mathrm{m}$. Plusieurs centaines d'entre eux ont été testés pour sélectionner ceux dont la qualité de diffraction permettra de déterminer un modèle atomique précis et de comprendre ainsi les interactions présentes dans ce complexe. L'évaluation de ces centaines d'échantillons est devenue possible à l'ESRF grâce à l'automatisation de ses lignes de lumière. Les données finales ont été collectées sur la ligne de lumière ID14-4.

Le modèle cristallin révèle une transmission complexe de l'information génomique à l'échelle atomique, du centre du ribosome jusqu'à sa surface en interaction avec FE-Tu [5]. La distance parcourue est d'environ $80 \AA$. Cette étude a aussi mis en évidence la présence de guanosine triphosphate (GTP), fournisseur d'énergie permettant l'entrée de l'acide aminé dans le site actif et sa liaison au polypeptide en cours d'élongation, quand le FE-Tu délivre l'ARN $N_{t}$ au ribosome. L'antibiotique utilisé pour l'obtention des cristaux étudiés bloque le complexe dans un état intermédiaire stable, dans lequel le GTP a été activé sans permettre une élongation de la chaîne polypeptidique.

\section{Mécanisme réactionnel de la PGK, ou l'art de combiner la haute et la basse résolution}

La production d'énergie a un rôle majeur dans la vie des organismes vivants. La glycolyse est la voie métabolique qui casse les sucres en produisant du $\mathrm{CO}_{2}$ de façon anaérobie. Cette voie est commune à tous les organismes vivants et serait aussi ancienne que la vie. Elle existait il y a trois milliards d'années, avant même l'accumulation d'oxygène dans l'atmos- phère, et constitue certainement le plus ancien mode d'obtention de l'énergie encore présent aujourd'hui. La phosphoglycérate kinase (PGK) catalyse la $7^{\text {e }}$ étape de cette voie, qui est l'étape réellement productrice d'énergie sous forme d'adénosine triphosphate (ATP), source universelle d'énergie dans un très grand nombre de réactions enzymatiques. Cette enzyme (la PGK) est donc placée à un poste clé de cette production d'énergie, ce qui en fait une cible potentielle, par exemple pour des médicaments contre les protozoaires anaérobies responsables de la maladie du sommeil et du paludisme. De plus, la PGK joue un rôle primordial dans la lutte contre le VIH et le virus de l'hépatite, en transférant in vivo un troisième groupement phosphate sur des molécules médicamenteuses qui, de ce fait, acquièrent une activité antirétrovirale. Pour toutes ces raisons, obtenir une image au niveau atomique de la fonction catalytique de cette enzyme serait une avancée scientifique notable pour la compréhension de son fonctionnement et pour la recherche de médicaments associés.

La PGK a une "structure en coquille ", comportant deux domaines structuraux indépendants reliés par une charnière, et peut se trouver sous deux formes, ouverte ou fermée (fig. 5a); chacun des deux domaines est lié à l'un des deux substrats nécessaires à la réaction, le glycérate et l'ADP/ATP. Des études cristallographiques antérieures ont défini l'état ouvert comme celui n'ayant aucune activité enzymatique, puisque les deux substrats sont alors trop éloignés. L'hypothèse d'un réarrangement moléculaire autour de la charnière liant les deux domaines, permettant le rapprochement des deux substrats et donc la réaction de phosphorylation (transfert d'un groupe phosphate du glycérate à l'ADP pour former de l'ATP) dans la forme fermée, avait été émise. Les structures de chacune des deux formes sont connues, mais restent des clichés figés d'une enzyme en continuel mouvement entre les états ouvert et fermé.

La combinaison des résultats de cristallographie, donnant une vue figée mais à très haute résolution, avec les résultats de diffusion des rayons $\mathrm{X}$ aux petits angles en solution (SAXS), à basse résolution $(\sim 15 \AA)$ mais dynamiques, deux techniques accessibles grâce au rayonnement synchrotron de l'ESRF, ont permis d'élucider le mécanisme réactionnel de cette protéine. Le SAXS permet une analyse des protéines en solution, plus proche de leur environnement naturel in vivo, et donne des renseignements sur l'enveloppe de la protéine et donc sur les mouvements relatifs d'un domaine par rapport à l'autre.

En premier lieu, la PGK a été étudiée en SAXS sous la forme ouverte, lorsqu'aucun substrat n'est fixé. La comparaison avec les données cristallographiques a montré qu'en solution, cette conformation était plus ouverte que dans les cristaux qui engendrent une forme de contrainte stérique. Ensuite, plusieurs intermédiaires réactionnels ont été analysés en solution et comparés avec les données cristallographiques. La plus grande surprise a été de voir que la protéine ne passe en moyenne que $7 \%$ de son temps en conformation fermée, catalytiquement active, et reste sous forme ouverte le reste du temps.

Afin de déterminer l'origine de la stabilité conformationnelle de la forme ouverte en solution, une étude numérique dynamique DEN (deformable elastic network [6]), utilisant comme modèle de départ les structures cristallographiques, a permis d'obtenir un modèle atomique des diverses formes de PGK en solution (fig. 5a). Ce modèle montre qu'une région hydrophobe est exposée à l'eau sous la forme fermée, alors qu'elle en est protégée sous la forme ouverte (fig. 5b). Ceci serait la cause primordiale de la prépondérance de la forme ouverte, puisque thermodynamiquement plus stable, même si la protéine est capable d'osciller entre les deux conformations. Cette surface hydrophobe jouerait donc le rôle de ressort, appliquant une force de résistance lorsque la protéine est sous forme fermée pendant la réaction catalytique, et permettant d'éjecter avec d'autant plus d'efficacité les produits une fois la réaction finie [7].

La combinaison de la cristallographie et du SAXS a donc permis de proposer un chemin réactionnel, et de visualiser au niveau atomique le passage de l'enzyme entre la forme ouverte en attente de substrat et la forme fermée durant laquelle a lieu la réaction enzymatique. 

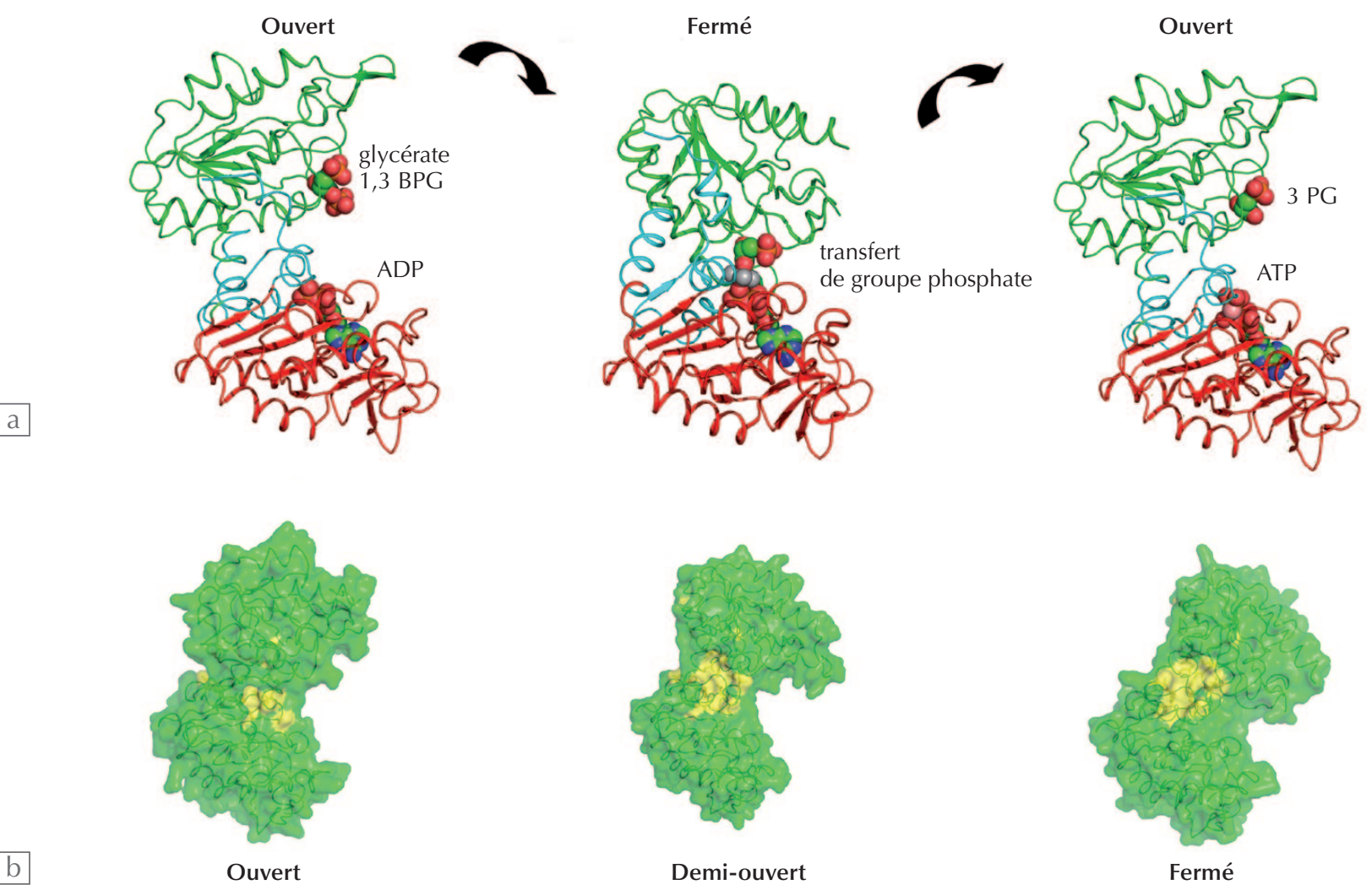

Demi-ouvert

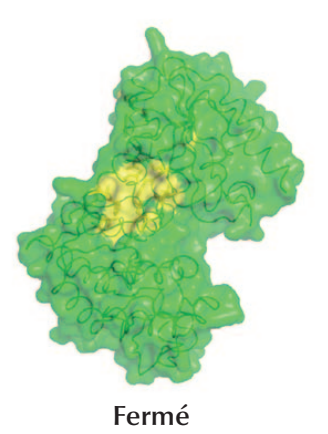

5. Mouvement structural de la PGK pendant la catalyse [7].

(a) À gauche : représentation schématique de la structure de l'état ouvert déterminée par DEN, en utilisant les données cristallographiques et de SAXS ; dans cette conformation, la PGK est capable de lier le substrat glycérate (1,3 BPG) sur son domaine vert et le cofacteur ADP sur son domaine rouge. Au centre : une rotation de $56^{\circ}$ autour de l'axe charnière (en bleu) rend possible la réaction de phosphorylation de l'ADP et la production d'ATP dans l'état fermé de la PGK. À droite : retour à l'état ouvert pour éjecter les produits de réaction ATP et 3 PG.

(b) La surface hydrophobe (représentée en jaune) joue le rôle de ressort thermodynamique régulant ainsi le mouvement structural et, par extension, la catalyse enzymatique. La représentation sous forme de surface montre que cette région devient progressivement exposée au solvant lors de la fermeture de l'enzyme (de gauche à droite).

\section{Conclusion}

Nous avons assisté ces dernières décennies à un engouement sans précédent pour la biologie structurale. Les centaines de projets financés par les organismes européens et nationaux en sont la preuve quotidienne. Les quelques exemples de recherches effectuées à l'ESRF présentés ci-dessus montrent à quel point le rayonnement synchrotron a contribué, et contribue encore à l'heure actuelle, à ce champ d'activité en pleine expansion. Cela est également démontré par les prix Nobel qui ont été remis en 2009 et 2012 pour les découvertes des structures du ribosome et d'une GPCR.

Nous pouvons imaginer qu'une telle dynamique puisse être poussée dans ses retranchements à plus ou moins long terme avec le programme de rénovation et de développement qu'a lancé l'ESRF, qui va permettre, grâce à l'automatisation, de tester jusqu'à 1000 cristaux par jour. Manifestement, ce programme demandera un grand effort en termes de développement de software et d'analyse de données sur les différentes lignes de lumière. Grâce à l'utilisation à grande échelle de robots issus de technologies industrielles, les dernières étapes manuelles de manipulation des cristaux seront éliminées, tout en maintenant un haut niveau de fiabilité. En combinant ces développements avec l'amélioration projetée de l'accélérateur de l'ESRF, les nouvelles lignes de lumière vont, à l'avenir, permettre d'obtenir des informations biologiques sur des systèmes toujours plus complexes. I (a) Agoniste : substance qui se fixe sur le même récepteur qu'une substance de référence et qui produit, au moins en partie, les mêmes effets.

(b) Expression d'une protéine : synthèse biochimique à partir du code génétique.

(c) Récepteur adrénergique : qui est activé par l'adrénaline.

(d) Peptide : chaîne d'acides aminés. Au-delà d'une dizaine d'acides aminés, on parle de polypeptide.

\section{Références}

1 R.E. Dickerson, Present at the flood: how structural molecular biology came about (Sinauer Associates, Sunderland, Mass., 2005).

2- Protein Data Bank: www.pdb.org/

3- ESRF Structural Biology Group: http://go.esrf.eu/MX

4• T. Warne et al., Nature 454 (2008) 486-492.

5• T.M. Schmeing et al., Science 326 (2009) 688-694.

6• G.F. Schröder et al., Nature 464 (2010) 1218-1222.

7• L. Zerrad et al., J. Biol. Chem. 286 (2011) 14040-14048. 